\title{
Height variation of the cutoff frequency in a sunspot umbra
}

\author{
T. Felipe ${ }^{1,2}$, C. Kuckein ${ }^{3}$, and I. Thaler ${ }^{4,5}$ \\ 1 Instituto de Astrofísica de Canarias, C/ Vía Láctea s/n, 38205 La Laguna, Tenerife, Spain \\ e-mail: tobias@iac.es \\ 2 Departamento de Astrofísica, Universidad de La Laguna, 38205 La Laguna, Tenerife, Spain \\ 3 Leibniz-Institut für Astrophysik Potsdam (AIP), An der Sternwarte 16, 14482 Potsdam, Germany \\ ${ }^{4}$ Kiepenheuer-Institut für Sonnenphysik, Schöneckstr. 6, 79104 Freiburg, Germany \\ 5 Racah Institute of Physics, The Hebrew University of Jerusalem, 91904 Jerusalem, Israel
}

Received 4 April 2018 / Accepted 15 June 2018

\begin{abstract}
Context. In the solar atmosphere, the acoustic cutoff frequency is a local quantity that depends on atmospheric height. It separates low-frequency evanescent waves from high-frequency propagating waves.

Aims. We measure the cutoff frequency of slow magnetoacoustic waves at various heights of a sunspot umbra and compare the results with the estimations from several analytical formulae.

Methods. We analyzed the oscillations in the umbra of a sunspot belonging to active region NOAA 12662 observed in the $10830 \AA$ spectral region with the GREGOR Infrared Spectrograph and in the Fe I 5435 A line with the GREGOR Fabry-Pérot Interferometer. Both instruments are attached to the GREGOR telescope at the Observatorio del Teide, Tenerife, Spain. We computed the phase and amplification spectra between the velocity measured from various pairs of lines that sample various heights of the solar atmosphere. The cutoff frequency and its height variation were estimated from the inspection of the spectra.

Results. At the deep umbral photosphere the cutoff frequency is around $5 \mathrm{mHz}$ and it increases to $6 \mathrm{mHz}$ at higher photospheric layers. At the chromosphere the cutoff is $\sim 3.1 \mathrm{mHz}$. A comparison of the observationally determined cutoff with the theoretically predicted values reveals an agreement in the general trend and a reasonable match at the chromosphere, but also significant quantitative differences at the photosphere.

Conclusions. Our analyses show strong evidence of the variation of the cutoff frequency with height in a sunspot umbra, which is not fully accounted for by current analytical estimations. This result has implications for our understanding of wave propagation, the seismology of active regions, and the evaluation of heating mechanisms based on compressible waves.
\end{abstract}

Key words. Sun: oscillations - Sun: photosphere - Sun: chromosphere - sunspots - techniques: polarimetric methods: observational

\section{Introduction}

The pionering work by Lamb (1909) showed that the cutoff frequency is a fundamental property of stratified mediums that determines the propagation of acoustic waves. Acoustic waves with frequencies above the cutoff can freely propagate in the atmosphere, whereas lower frequencies lead to non-propagating waves, also known as evanescent waves. Lamb's original work studied an isothermal atmosphere. In this case, the cutoff frequency is a global property and it is the same in the whole atmosphere. In the general case of a non-isothermal medium, such as the situation of the solar atmosphere, the cutoff frequency is a local quantity that depends on the atmospheric height.

Several works have attempted to derive an expression for the cutoff frequency in non-isothermal atmospheres (e.g., Schmitz \& Fleck 1998; Deubner \& Gough 1984; Musielak et al. 2006). However, various formulae for the cutoff have been obtained depending on the selection of dependent and independent variables for the wave equations (Schmitz \& Fleck 2003). The situation described above does not take into account magnetic fields, which modify not only the existing temperature and pressure stratification, but also introduce new types of waves. The cutoffs of magnetohydrodynamic (MHD) waves in isothermal atmospheres with uniform magnetic field (e.g., Thomas 1982, 1983; Campos 1986; Stark \& Musielak 1993; Roberts 2006) and magnetic fluxtubes (e.g., Rae \& Roberts 1982; Roberts 1983; Hammer et al. 2010; Murawski \& Musielak 2010; Routh et al. 2013; Perera et al. 2015) have been thoroughly explored. Using the Solar Optical Telescope aboard Hinode spacecraft, Fujimura \& Tsuneta (2009) measured the phase differences between line-of-sight (LOS) velocity and magnetic flux in pores and intergranular magnetic structures, and concluded that the observations are consistent with standing sausage and/or kink MHD waves.

This paper focuses on the analysis of oscillations in larger solar magnetic structures. In the presence of strong magnetic fields, $p$-modes traveling from the interior to the surface convert into slow and fast magnetoacoustic waves at the layer where the Alfvén speed and sound speed are similar (Cally 2006, 2007; Schunker \& Cally 2006; Khomenko \& Collados 2006). The efficiency of the conversion to each of these modes depends on the angle between the wave vector and magnetic field. In the region where the Alfvén speed is larger than the sound speed, the slow magnetoacoustic wave behaves like a sound wave, but it is guided along the field lines. This means that its velocity pertubations are along the field lines, while the wave can propagate in every direction except perpendicular to the 
magnetic field. Similar to non-magnetic acoustic waves, in a gravitationally stratified medium they are affected by the cutoff. However, their cutoff value is reduced by a factor $\cos \theta$, where $\theta$ is the field inclination from the solar vertical due to the reduced gravity along the magnetic field (Bel \& Leroy 1977; McIntosh \& Jefferies 2006; Jefferies et al. 2006). This ramp effect allows the detection of low-frequency compressive waves in the chromosphere and corona of regions with strong magnetic fields (Orrall 1966; Giovanelli et al. 1978; Lites et al. 1993; De Moortel et al. 2002; De Pontieu et al. 2004; Vecchio et al. 2007; Bloomfield et al. 2007), and has been employed to reconstruct the magnetic field inclination in sunspots (Tziotziou et al. 2006; Yuan et al. 2014; Löhner-Böttcher et al. 2016). An alternative process that leads to a reduction of the cutoff frequency is radiative energy losses (Roberts 1983; Centeno et al. 2006; Khomenko et al. 2008), although the numerical simulations from Heggland et al. (2011) indicate that field inclination is more relevant than radiation to explain long-period wave propagation.

In this work, we quantify observationally the acoustic cutoff frequency in a sunspot umbra. We focus on the analysis of slow magnetoacoustic waves propagating along vertical magnetic fields. The height variation of the cutoff is determined from multi-line observations. This paper is organized as follows. The observations and methods are described in Sect. 2. The analysis and results are presented in Sect. 3 and discussed in Sect. 4. Finally, conclusions are given in Sect. 5.

\section{Sunspot observations and data analysis}

The sunspot in active region NOAA 12662 was observed with the $1.5 \mathrm{~m}$ GREGOR solar telescope (Schmidt et al. 2012) on 2017 June 17. The sunspot was located at the solar position $x=-387^{\prime \prime}, y=179^{\prime \prime}(\mu=0.91$, with $\mu$ defined as the cosine of the heliocentric angle). Spectropolarimetric data was acquired with the GREGOR Infrared Spectrograph (GRIS; Collados et al. 2012) around the $1 \mu \mathrm{m}$ spectral window. The relevant lines include the photospheric Si I $10827 \AA$ and CaI $10839 \AA$ lines, together with the chromospheric He I $10830 \AA$ triplet and two telluric lines. The later two lines were used for the wavelength calibration on an absolute scale (Martínez Pillet et al. 1997; Kuckein et al. 2012). The slit (63". 5) was roughly fixed at the center of the sunspot and covered the umbra, penumbra, and granulation on both sides. The full-Stokes spectra were taken with an exposure time of $100 \mathrm{~ms}$ and ten accumulations. A total amount of 802 spectra were taken between 08:05 and 09:22 UT. The data reduction included dark and flat-field corrections and the standard polarimetric calibration (Collados 1999, 2003). The data for the polarimetric calibration were obtained with the GREGOR polarimetric calibration unit (Hofmann et al. 2012).

A second instrument, the GREGOR Fabry-Pérot Interferometer (GFPI; Puschmann et al. 2012, and references therein), was simultaneously acquiring spectroscopic images of the sunspot in the photospheric non-splitting Fe I $5435 \AA$ line. Each scan along the line consisted of 20 wavelength positions with eight accumulations per position and an exposure time of $20 \mathrm{~ms}$ each. The cadence was about $23 \mathrm{~s}$ in the $2 \times 2$ binned mode. The image scale was $0^{\prime \prime} .081 \mathrm{pixel}^{-1}$. The sunspot was placed in the center of the field of view (FOV), which had a size of $55^{\prime \prime} .7 \times 41^{\prime \prime} .5$. Observations with the GFPI started at 08:10 and stopped at 09:20 UT. In this period of time, a total amount of 184 scans

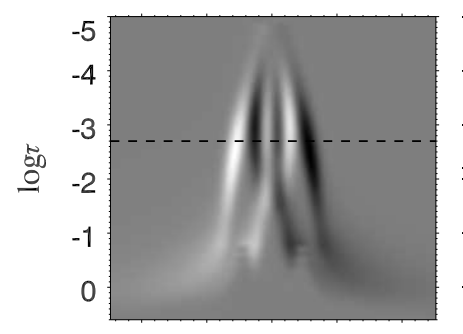

$\begin{array}{lllll}-1.0 & -0.5 & 0.0 & 0.5 & 1.0\end{array}$ $\Delta \lambda(\AA)$

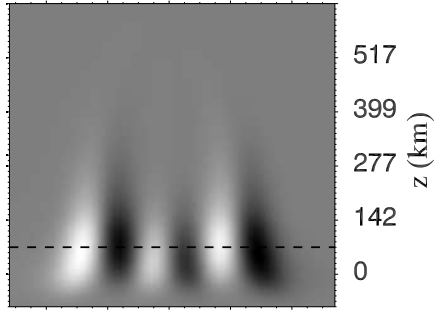

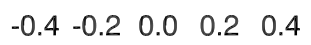
$\Delta \lambda(\AA)$
Fig. 1. Response functions of the intensity to the velocity of the Si I $10827 \AA$ (left panel) and Ca I $10839 \AA$ (right panel) lines in the average umbral atmosphere. Horizontal dashed lines indicate the optical depth selected for the analysis of the velocity from each line.

were taken. The data reduction was accomplished using sTools (Kuckein et al. 2017). The pipeline carries out dark, flat-field, and prefilter curve corrections. The blueshift across the FOV was removed. The broadband and narrowband images were restored using multi-object multi-frame blind deconvolution (MOMFBD; Löfdahl 2002; van Noort et al. 2005). However, sporadic periods of poor seeing spoiled our long time series producing several incorrectly restored images. Therefore, to assure a continuous analysis of the oscillations, we only focused on the non-restored data.

For the photospheric lines (Si I $10827 \AA$ and Ca I $10839 \AA$ ), the temporal evolution of the sunspot atmosphere was determined using the SIR code (Ruiz Cobo \& del Toro Iniesta 1992). For each spatial position and time step, SIR provides the vertical stratification of the magnetic and thermodynamic atmospheric parameters retrieved from a process in which an initial guess atmosphere (in our case, the cool sunspot model from Collados et al. 1994) is iteratively modified until it reproduces the observed Stokes parameters. We performed independent single-component inversions for the Si I $10827 \AA$ and Ca I $10839 \AA$ lines. In both cases, the temperature stratification is modified with three nodes, while the other physical parameters (LOS velocity, microturbulence, field strength, and inclination, and azimuth angles of the magnetic field) are changed with two nodes. In this paper, we focus on the analysis of the LOS velocities in the sunspot umbra. We selected the optical depth for which the response of the lines to the velocity is maximum (see Fig. 1). In the case of the Ca I $10839 \AA$ line, this value corresponds to $\log \tau \sim-0.5$, while for the Si I $10827 \AA$ it is $\log \tau \sim-2.7$. In the following, we refer to those optical depths when we discuss Ca I $10839 \AA$ and Si I $10827 \AA$ LOS velocities. The response functions illustrated in Fig. 1 were computed for the model obtained as the average of atmospheres retrieved from the inversion of the Si I $10827 \AA$ line in the umbra. In this model, the maximum response of the Ca I $10839 \AA$ and Si I $10827 \AA$ lines to the velocity corresponds to a geometrical height of 64 and $340 \mathrm{~km}$, respectively, with $z=0$ located at the optical depth where $\log \tau=0$. The top panels of Fig. 2 illustrate the velocity maps of these two lines. The LOS velocity from the chromospheric He I $10830 \AA$ line (Fig. 2d) has been retrieved from a Milne-Eddington inversion using the code MELANIE (Socas-Navarro 2001). Figure 3 shows the temporal evolution of the LOS velocities at a selected umbral location where the strongest He I $10830 \AA$ velocity oscillations are detected. Around $t=16 \mathrm{~min}$ its peak-to-peak amplitude is 

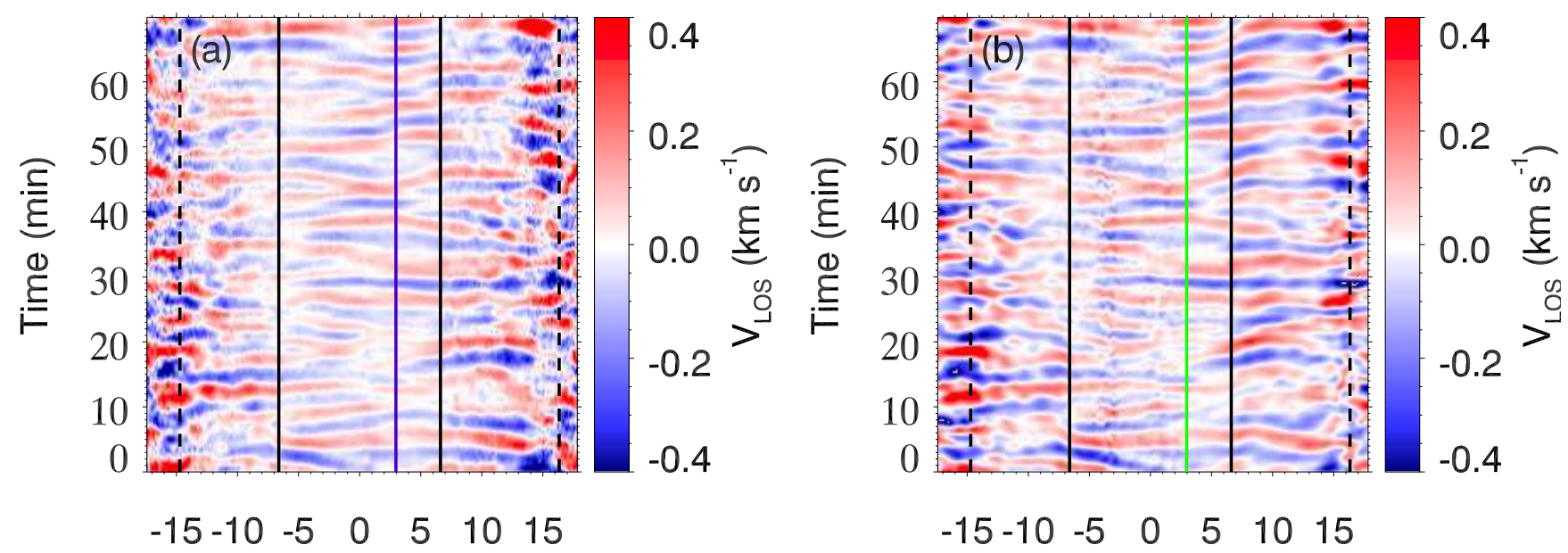

\section{Horizontal distance $(\operatorname{arcsec})$}
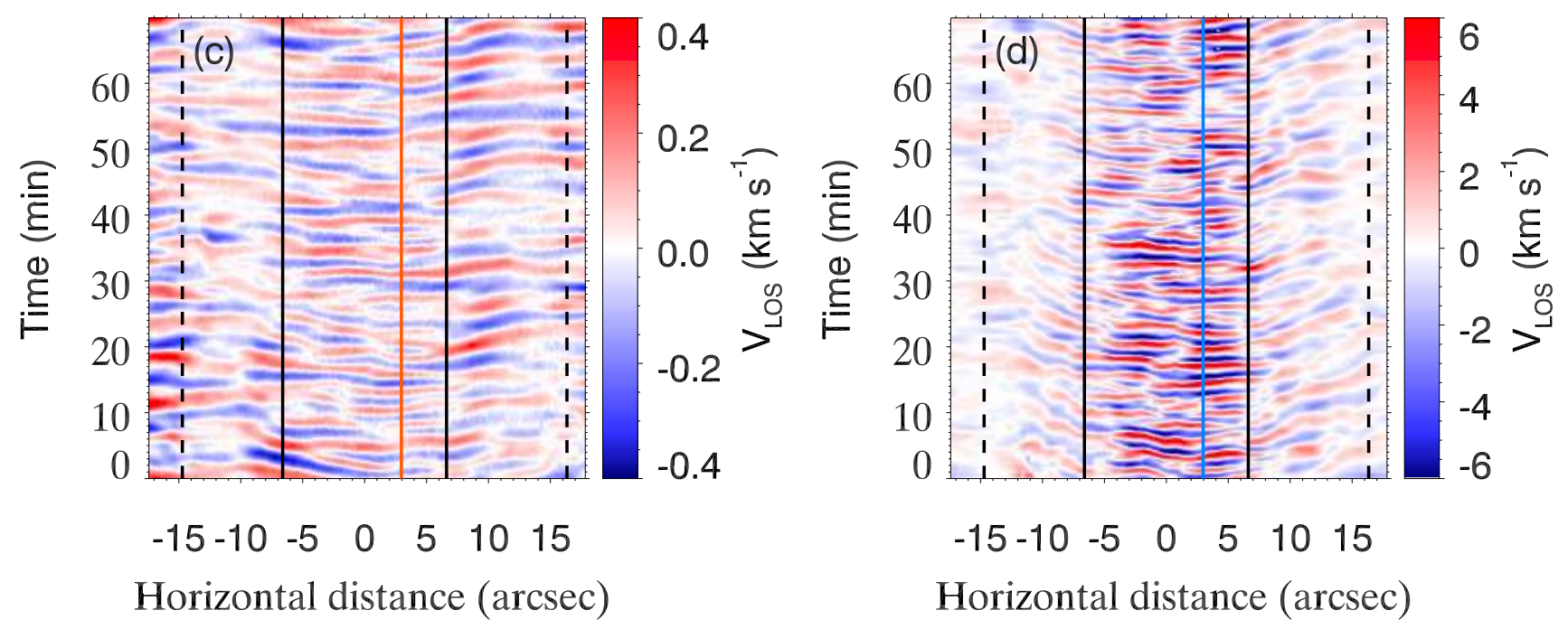

Fig. 2. LOS velocity measured with the Ca I $10839 \AA$ line (panel a), Si I $10827 \AA$ line (panel b), Fe I $5435 \AA$ line (panel c), and He I $10830 \AA$ triplet (panel d). The signals have been filtered in the frequency range between 1 and $15 \mathrm{mHz}$. Positive velocities (red) correspond to downflows and negative velocities (blue) to upflows. The vertical colored lines indicate the umbral location illustrated in Fig. 3.

$22 \mathrm{~km} \mathrm{~s}^{-1}$, which is significantly larger than the amplitude of any previously detected umbral oscillations in He I $10830 \AA$ (generally below $14 \mathrm{~km} \mathrm{~s}^{-1}$; e.g., Centeno et al. 2006; Felipe et al. 2010).

The Fe I $5435 \AA$ line observed with GFPI is formed at high photospheric layers. Using NLTE modeling of the spectral line radiative transfer and numerical simulations of granular convection, Bello González et al. (2010a) estimated a formation height of $500 \mathrm{~km}$ in granules and $620 \mathrm{~km}$ in intergranules. Assuming that in the sunspot umbra the response function of the line to the velocity peaks at the same density as in intergranules, the formation height of the Fe I $5435 \AA$ line in our average umbra is $510 \mathrm{~km}$. The LOS velocity of the Fe I $5435 \AA$ line was determined by measuring the Doppler shift using a second order polynomial fit to the intensity core of the line. The Fe I $5435 \AA$ line is insensitive to the magnetic field (Landé factor $g=0$ ) and, thus, the estimation of the core of the line is not affected by Zeeman splitting. We note that GFPI data provides the temporal evolution in a bidimensional surface. The following approach has been carried out in order to construct GFPI temporal series co-spatial to the location of the GRIS slit. First, Fe I $5435 \AA$ velocities from GFPI were converted to a new grid with the spatial size and the time steps of the GRIS data. Only the temporal span with simultaneous data acquisition in both instruments (between 08:10 and 09:20 UT) was used. Second, the GRIS Ca I $10839 \AA$ and the GFPI Fe I $5435 \AA$ velocities were filtered in the frequency band between 1.5 and $3 \mathrm{mHz}$. This way, we eliminate the propagating high-frequency waves (above the expected cutoff frequency) and keep only the evanescent waves. Third, assuming that the GRIS slit is aligned with the $X$ axis of the GFPI FOV, we iteratively computed the cross-correlation between the filtered Ca I $10839 \AA$ waves and the filtered Fe I $5435 \AA$ waves at different positions in the $Y$ direction and applying different shifts in the $X$ direction. Since those waves are evanescent, they oscillate in phase for different heights. We determined the location of the GRIS slit in the GFPI FOV as the $Y$ coordinate and the $X$ shift that shows the highest correlation. The position of the slit on a region of the GFPI FOV is illustrated in Fig. 4. Finally, that region has been extracted from the remapped temporal series obtained at the first step. Figure $2 \mathrm{c}$ shows the temporal evolution of the Fe I $5435 \AA$ velocity at the location of the GRIS slit. 

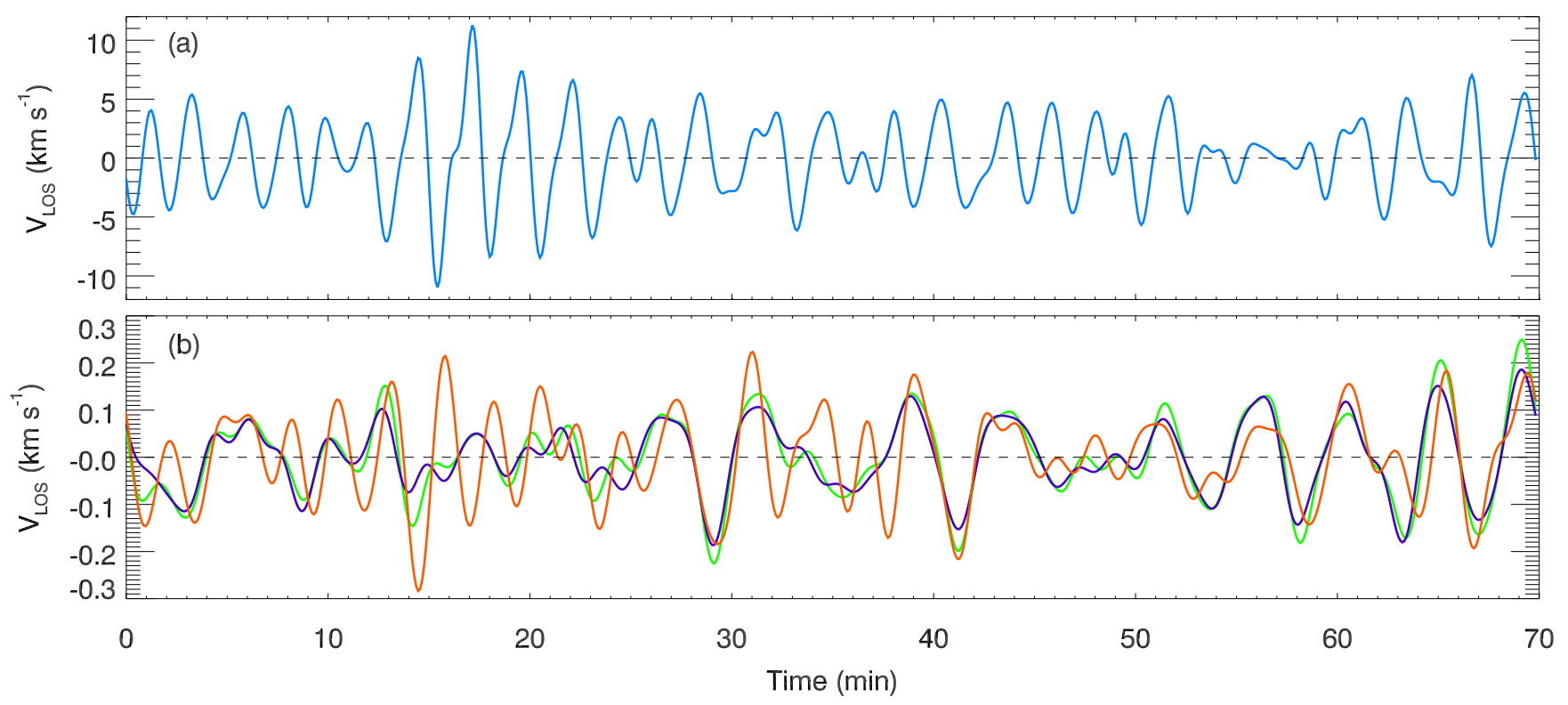

Fig. 3. Top panel: temporal evolution of the LOS velocity at a selected umbral location measured with the He I $10830 \AA$ triplet. Bottom panel: temporal evolution of the LOS velocity at the same umbral location measured with the Ca I $10839 \AA$ (violet), Si I $10827 \AA$ (green), and Fe I $5435 \AA$ (orange) lines. The umbral location is indicated by the vertical colored lines in Fig. 2.

The data from 2017 June 17 were complemented with another data set from 2007 August 28 obtained with the Vacuum Tower Telescope at the Observatorio del Teide. These data correspond to the active region NOAA 10969, which was located near disk center $(\mu=0.96)$, and the spectra were obtained from a slit placed over the center of a sunspot. These data were described and analyzed in Felipe et al. (2010). In this work, we use Doppler velocities of the Ca II H and He I $10830 \AA$ lines in order to include the analysis of two chromospheric lines obtained simultaneously.

\section{Results}

\subsection{Power spectra}

Figure 5 shows the umbral power (averaged for all the umbra, which is delimited by the black solid vertical lines in Fig. 2) of the LOS velocity of the four spectral lines from the 2017 June 17 dataset. As expected, most of the power of the photospheric Si I $10827 \AA$ (green) and Ca I $10839 \AA$ (violet) lines is concentrated in the $5 \mathrm{~min}$ band in the frequency band between 2 and $4 \mathrm{mHz}$. At the higher formation height of the Fe I $5435 \AA$ line (orange) the power in the $5 \mathrm{~min}$ band is still significant, but the strongest peak is found at $5.7 \mathrm{mHz}$. At the chromosphere (He I $10830 \AA$, blue line) the power spectra shows a broad distribution that has several power peaks in the frequency range between 5 and $9 \mathrm{mHz}$ ( $3 \mathrm{~min}$ band). The maximum power is located also at $5.7 \mathrm{mHz}$.

Interestingly, all the oscillatory signals, from the deep photosphere (Ca I $10839 \AA$ ) to the chromosphere (He I $10830 \AA$ ), exhibit power peaks at some shared frequencies. However, the relative power between these power peaks depends on the formation height of the line. The main chromospheric peaks (5.7, $6.4,7.4,8.1$, and $8.6 \mathrm{mHz}$ ) are already present in the velocity oscillations measured with the Ca I $10839 \AA$ and Si I $10827 \AA$ lines, although their photospheric power is insignificant in comparison with that in the $5 \mathrm{~min}$ band. This finding supports the results from Centeno et al. (2006), who proposed that the

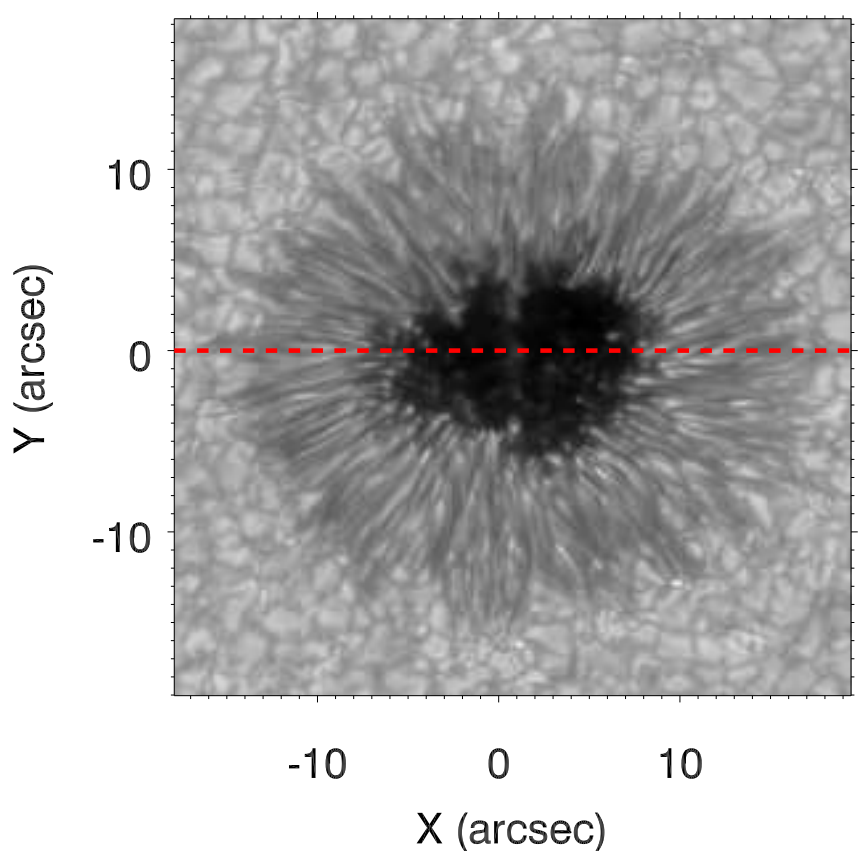

Fig. 4. Reconstructed broadband image of the sunspot NOAA 12662 on June 172017 at 08:12:45 UT from GFPI. The red dashed line indicates the location of the GRIS slit determined following the method described in Sect. 2.

chromospheric 3 min power reaches the higher atmospheric layers through wave propagation from the photosphere. The amplitude of the propagating high-frequency waves (above the cutoff frequency) increases more than that of the non-propagating (evanescent) low-frequency waves. This way, waves in the $3 \mathrm{~min}$ band dominate at chromospheric heights. Taking the former into account, an approximate value of the cutoff frequency can be estimated from an examination of the height variation of the power. For the Ca I $10839 \AA$ line (deep photosphere), the power at $5.2 \mathrm{mHz}$ is larger than the power at any higher frequency. 


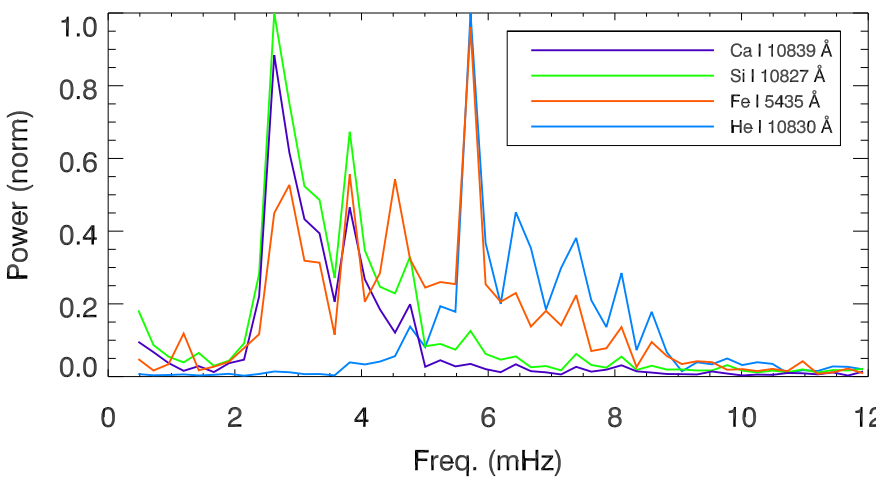

Fig. 5. Average umbral power spectra of the LOS velocities obtained with the Ca I $10839 \AA$ (violet), Si I $10827 \AA$ (green), Fe I $5435 \AA$ (orange), and He I $10830 \AA$ (blue) lines. The power of the three former lines is normalized to the maximum power of the SiI $10827 \AA$ line, while the He I $10830 \AA$ is normalized to its maximum power.

Although it increases with height, the increase rate of higher frequencies is larger. For example, for the Si I $10827 \AA$ line the power at the peak located $5.7 \mathrm{mHz}$ is already larger than the power peak at $5.2 \mathrm{mHz}$, and $5.7 \mathrm{mHz}$ oscillations dominate the high photosphere (Fe I $5435 \AA$ ) and chromosphere (He I 10830 $\AA$ ). This fact indicates that the cutoff frequency of the umbral atmosphere is around $5.5 \mathrm{mHz}$. However, the cutoff is not a constant quantity, since it changes with the atmospheric stratification. In the following section we explore the variation of the local cutoff values.

\subsection{Phase difference and cutoff}

We computed the phase difference $(\Delta \phi)$ as a function of frequency between velocity signals at two different heights for several pairs of lines. As a first example, Fig. 6 illustrates the phase difference between the two lines with larger difference in formation height, the CaI $10839 \AA$ and He I $10830 \AA$ lines. The phase difference at each spatial position was obtained by subtracting the phase of the former line to the phase of the latter. Thus, a positive (negative) value indicates upward (downward) wave propagation. The $2 \pi$ indetermination in the phase difference was manually unwrapped by adding $2 \pi$ to the $\Delta \phi$ negative values at high frequencies to maintain the smooth trend of $\Delta \phi$ measured at lower frequencies. The black solid line in the top panel of Fig. 6 shows the average of the phase difference inside the umbra. For each frequency, a histogram with the relative occurrence of a given value of $\Delta \phi$ (in 0.3 rad bins) over all pixels in the umbra with a magnetic field inclination below $20^{\circ}$ (in the local solar frame, according to the inversions of the Si I $10827 \AA$ line) has been computed. Only the spatial points whose $\Delta \phi$ is in a bin with more than $7 \%$ occurrence are included in the average. This way, we avoid contamination from outliers. The bars in the phase difference correspond to the standard deviation of the averaged points.

Following Centeno et al. (2006), we also computed the coherence and amplification spectra. The coherence depends on the frequency and assumes values in the range [0,1]. A higher coherence indicates that the phase difference between two signals is characteristic of their oscillations. We consider the phase difference to be significant for coherence values above 0.7 . The amplification spectra is defined as the ratio between the power of the two signals, averaged in the umbra. The bottom panel of

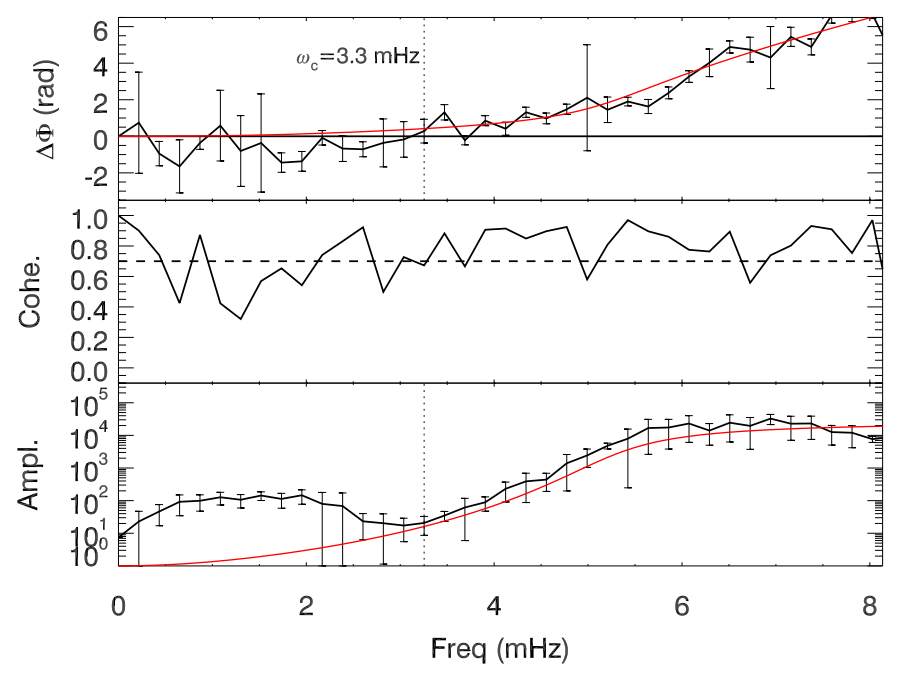

Fig. 6. Phase (top panel), coherence (middle panel), and amplification (bottom panel) spectra between the LOS velocities of the Ca I $10839 \AA$ and He I $10830 \AA$. Black line indicates the measured values and the red lines correspond to the best fit to a theoretical model. Errors are determined as described in the text.

Fig. 6 (and the other figures with the same format) depicts the standard deviation of the amplification for the umbral points.

The phase and amplification spectra were fitted to a theoretical model of slow mode wave propagation in a gravitationally stratified isothermal atmosphere that allows radiative losses (see Centeno et al. 2006; Felipe et al. 2010, for details). The fitting is performed manually with three free parameters: atmospheric temperature $(T)$, height difference between the formation height of the two lines $(\Delta z)$, and the characteristic timescale of the radiative losses $\left(\tau_{\mathrm{R}}\right)$. With the inclusion of the radiative losses in the modeling, there is not a strict cutoff frequency that separates propagating (high-frequency) waves from non-propagating (evanescent, low-frequency) waves. Instead, all frequencies are propagated and reflected simultaneously and the ratio of propagation versus reflection grows with frequency. Although there is no cutoff frequency, an effective cutoff frequency, separating mainly propagating waves from mainly non-propagating waves, can be defined. The parameters determined from this fitting for all the analyzed pairs of lines are shown in Table 1.

Figure 6 indicates that propagating waves go upward in the umbral atmosphere. The examination of the phase and amplification spectra allow us to estimate the cutoff frequency. For frequencies with $\Delta \phi$ around $0^{\circ}$ and a low amplitude increase, the observed oscillations correspond to evanescent waves. On the contrary, frequencies with $\Delta \phi>0^{\circ}$ and a larger amplitude increase are indicative of upward propagating waves. However, the solar atmosphere is a stratified and inhomogenous medium and, thus, the cutoff frequency is a local quantity that depends on the atmospheric height. We probed the height dependence of the cutoff frequency by estimating it for several pairs of lines. We defined the observed cutoff frequency $\omega_{\mathrm{c}}$ as the highest frequency whose phase difference is not undoubtedly different from zero. In order to ensure this condition, for each pair of lines we selected the highest frequency with $\Delta \phi$ and its standard deviation out of a value of zero and with a coherence higher than the chosen confidence threshold of 0.7 . In addition, we verified that the increasing trend of the phase difference is maintained for the following frequency values and that this increasing phase difference is accompanied by an increase in the amplification spectra. 
Table 1. Parameters for phase and amplification spectra fits and observed cutoff frequency.

\begin{tabular}{ccccc}
\hline \hline Line pair & $T(\mathrm{~K})$ & $\Delta z(\mathrm{~km})$ & $\tau_{\mathrm{R}}(\mathrm{s})$ & $\omega_{\mathrm{c}}(\mathrm{mHz})$ \\
\hline Ca I-Si I & 4000 & 130 & 30 & 5.0 \\
Si I-Fe I & 3000 & 200 & 20 & 6.0 \\
Fe I-He I & 3500 & 750 & 40 & 3.6 \\
Ca II H-He I & 7000 & 150 & 20 & 3.1 \\
Ca I-He I & 4000 & 1100 & 40 & 3.3 \\
Si I-He I & 4000 & 1000 & 40 & 3.3 \\
\hline
\end{tabular}

Notes. The first column indicates the pair of lines used in the analysis; the second to fourth columns are the temperature $(T)$, difference in formation heights $(\Delta z)$, and characteristic timescale of the radiative losses $\left(\tau_{\mathrm{R}}\right)$ retrieved from the fit of the phase and amplification spectra to a model of linear wave propagation in a gravitationally stratifed atmosphere with radiative losses; and the last column is the observationally measured cutoff frequency $\left(\omega_{\mathrm{c}}\right)$.

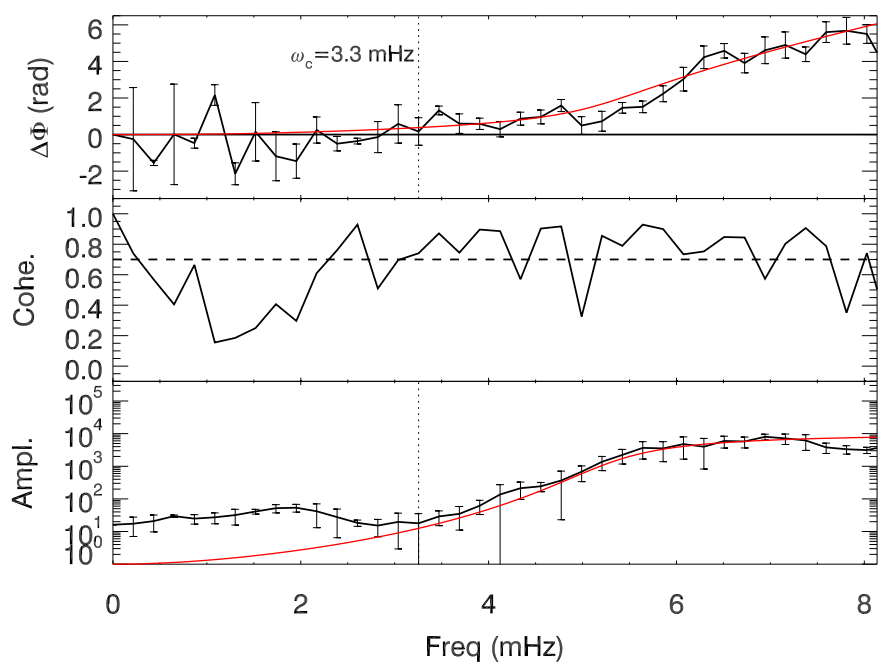

Fig. 7. Same as Fig. 6 but for the line pair Si I $10827 \AA$ and He I $10830 \AA$.

The observationally estimated cutoff frequencies are shown in the last column of Table 1 . These frequencies were determined from the measurements illustrated in Figs. 6-11. The analysis of pairs of photospheric and chromospheric lines (Ca I $10839 \AA$ A-He I $10830 \AA$ A, Si I 10827 A-He I $10830 \AA$ A, Figs. 6 and 7, respectively) shows a similar behavior. For low frequencies, the phase difference is around zero and has noisy measurements and low coherence. For frequencies above $3.3 \mathrm{mHz}$, the phase and amplification spectra start to progresively increase, indicating upward wave propagation. This value was chosen as the cutoff frequency, since it is evident that waves with higher frequencies have propagated at least at some heights of the umbral atmosphere. We note that this cutoff value must be interpreted as the minimum value of the cutoff between the formation height of the photospheric and chromospheric lines. Even though between these two layers waves can become evanescent in some regions, we can detect the phase difference produced in the heights where waves were able to propagate. We sampled the atmospheric variation of the cutoff value by exploring pairs of lines with smaller differences in formation height. Thus, these pairs of lines sample the cutoff at localized layers of the atmosphere.

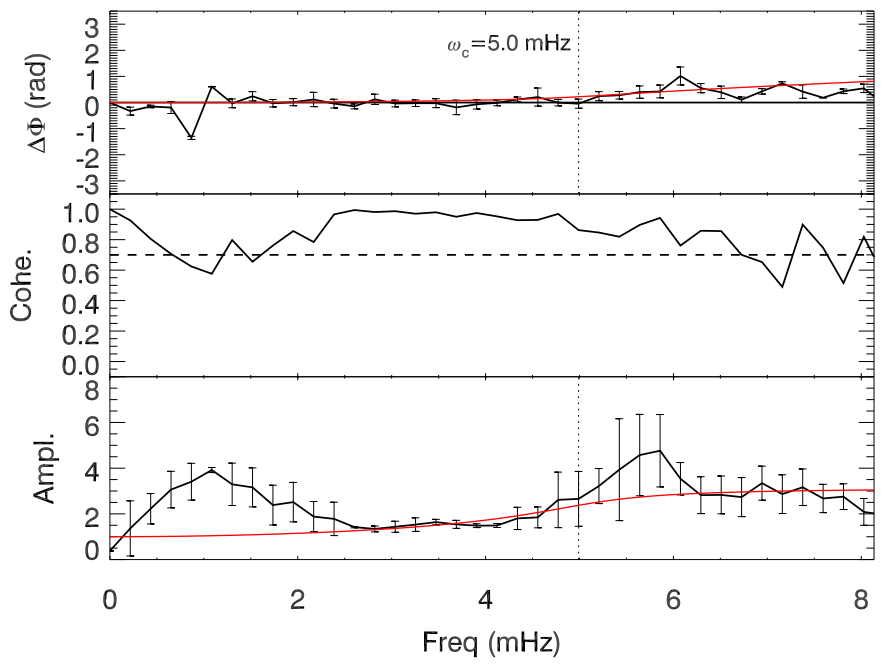

Fig. 8. Same as Fig. 6 but for the line pair Ca I $10839 \AA$ and Si I $10827 \AA$.

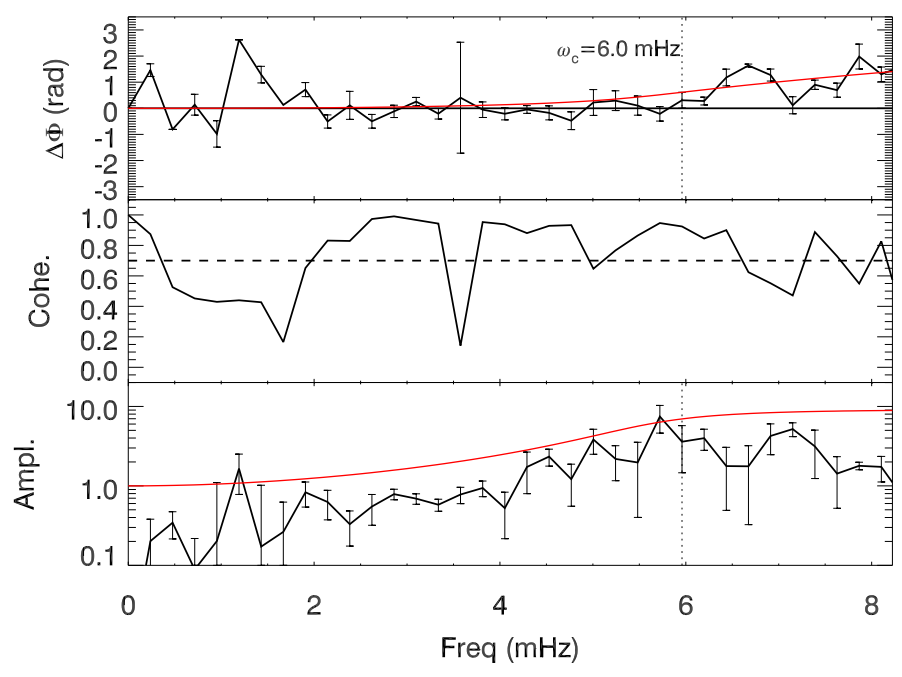

Fig. 9. Same as Fig. 6 but for the line pair Si I $10827 \AA$ and Fe I $5434.534 \AA$.

Figure 8 illustrates the results for the Ca I $10839 \AA$-Si I $10827 \AA$ pair, that is, for wave propagation between the deep photosphere and medium photosphere. Owing to the small height difference between the formation height of these two lines, the values of $\Delta \phi$ are low, below $1 \mathrm{rad}$. Phase differences different from zero are detected for frequencies higher than $5.0 \mathrm{mHz}$. This cutoff value must be interpreted as an upper limit of the actual cutoff. Waves with lower frequencies could propagate, but their low phase difference (due to the small difference in formation height between both lines) might be undetectable for the uncertanties of our measurements. The amplification spectra seems to point to that, since an increase in the amplification is found for frequencies above $4.1 \mathrm{mHz}$, although this trend is also masked by the errors of the amplification spectra.

Waves between the photosphere (Si I $10827 \AA$ ) and high photosphere (Fe I $5435 \AA$ ) are analyzed in Fig. 9. The phase difference spectra exhibits a cutoff frequency of $6.0 \mathrm{mHz}$ although, similar to the previous case, the amplification spectra points to wave propagation for lower frequencies. Interestingly, the undoubtedly evanescent waves (frequencies below $4 \mathrm{mHz}$ ) show 


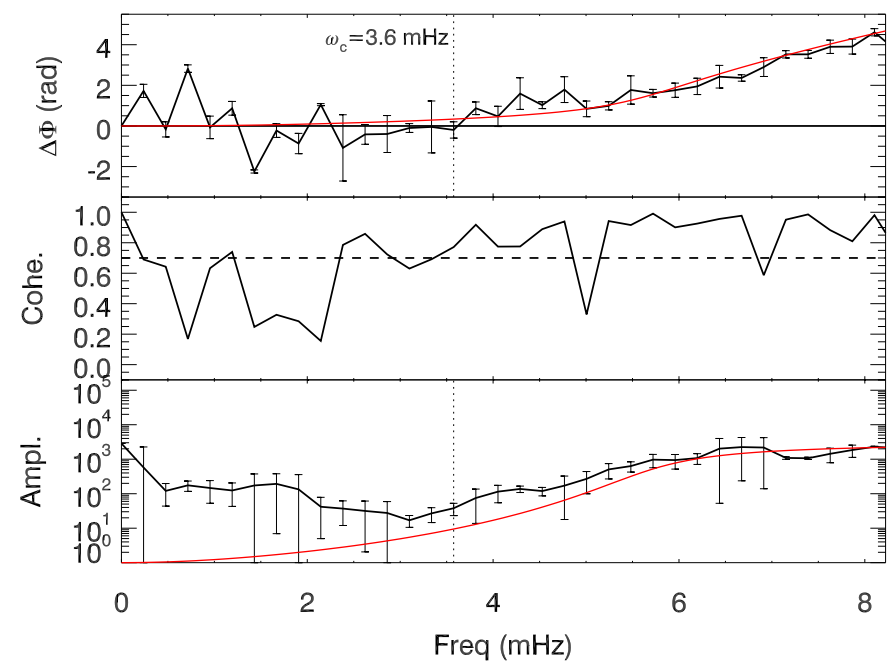

Fig. 10. Same as Fig. 6 but for the line pair Fe I $5434.534 \AA$ and He I $10830 \AA$.

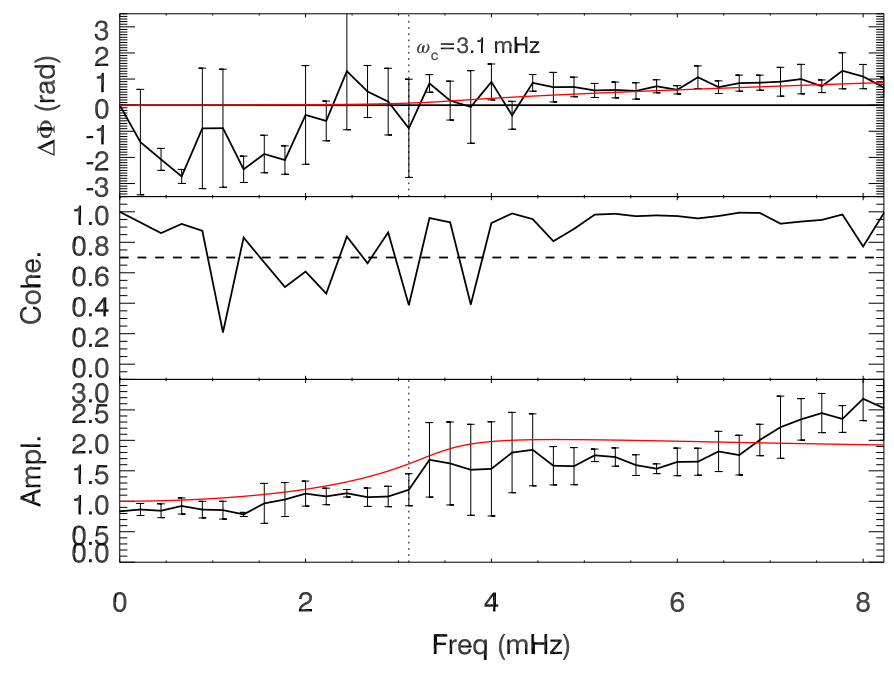

Fig. 11. Same as Fig. 6 but for the line pair Ca II H and He I $10830 \AA$ A.

lower amplitude at the formation height of the Fe I $5435 \AA$ line than at the Si I $10827 \AA$ line. This is consistent with the power spectra illustrated in Fig. 5, but cannot be understood from a wave model (see red line in the bottom panel of Fig. 9). We consider that this reduction in the velocity amplitude of the Fe I $5435 \AA$ line is due to the different acquisition strategy, since the data from the Fabry-Pérot interferometer has lower spectral resolution than that from the slit spectrograph and it requires more time to scan the spectral profile of the line.

Figure 10 shows that waves with frequency above $3.6 \mathrm{mHz}$ can travel from the high photosphere (Fe I $5435 \AA$ ) to the chromosphere (He I $10830 \AA$ ). In this case, the estimation of the cutoff frequency from the phase difference spectra is in agreement with that from the amplification spectra. Finally, the phase difference between the chromospheric Ca II H and He I $10830 \AA$ lines (Fig. 11) shows continuous positive phase shifts for frequencies above $4.2 \mathrm{mHz}$, while lower frequencies $(3.3$ and $4 \mathrm{mHz}$ ) also exhibit a reliable positive phase difference. Based on these data and the variation found for the amplification spectra, we determine a cutoff frequecy of $3.1 \mathrm{mHz}$.

\section{Discussion}

The cutoff frequency is a well-known property of gravitationally stratified atmospheres. Despite its strong impact on wave propagation, there is not a clear definition of its value, since the analytical expression obtained for its computation depends on the choice of the dependent and independent variables used in the wave equation (Schmitz \& Fleck 2003). Only in the case of an isothermal atmosphere can a correct formula for the cutoff be determined as (Lamb 1909)

$\omega_{\mathrm{C} 1}=\frac{c_{\mathrm{s}}}{2 H_{\mathrm{p}}}$,

where $c_{\mathrm{s}}$ is the sound speed and $H_{\mathrm{p}}$ is the pressure scale height. However, the solar atmosphere is not isothermal, and this expression is not generally applicable. The most commonly form used by helioseismologists is

$\omega_{\mathrm{C} 2}=\frac{c_{\mathrm{s}}}{2 H_{\rho}}\left(1-2 \frac{\mathrm{d} H_{\rho}}{\mathrm{d} z}\right)^{1 / 2}$,

where $H_{\rho}$ is the density scale height. This expression produces a sharp spike just below the solar surface, which is inconsistent with the Wentzel, Kramers, and Brillouin (WKB) approximation that is usually employed for the study of linear acoustic waves. This approximation is only valid for waves with wavelengths shorter than the characteristic scales of the atmosphere. Some studies using the WKB approximation use the isothermal expression (Eq. (1)), even for non-isothermal problems (Cally 2007; Moradi \& Cally 2008). The WKB approximation has also been used to derive a local dispersion relation and cutoff frequency for MHD waves in an isothermal solar atmosphere (Thomas 1982, 1983; Campos 1986). In addition, in models with radiative losses there is no clear cutoff frequency, but a pseudo-cutoff can be defined (Centeno et al. 2006). This effective cutoff can be significantly lower than that obtained for the adiabatic case.

Recently, Wiśniewska et al. (2016) have estimated the cutoff frequency of the solar quiet-Sun atmosphere (including its variation with the atmospheric height) and compared the results with the formulae derived from various theories. In this paper we aim to perform a similar estimation for a sunspot umbra atmosphere. In addition to Eqs. (1) (assuming that it changes locally) and (2), we also evaluated the expression derived by Schmitz \& Fleck (1998), i.e.,

$\omega_{\mathrm{C} 3}=\omega_{\mathrm{C} 1}\left(1-2 \frac{\frac{\mathrm{d} c_{\mathrm{s}}}{\mathrm{d} z}}{\omega_{\mathrm{C} 1}}\right)^{1 / 2}$.

The above formulae were derived for acoustic waves and do not take into account the presence of the magnetic field. However, we expect these formulae to be applicable to the slow magnetoacoustic waves analyzed in this work, since they have an acousticlike behavior; we are observing an umbral atmosphere region in which the Alfvén speed $v_{\mathrm{A}}$ is higher than the sound speed $c_{\mathrm{s}}$. We also explored a specific formula for cutoff frequencies obtained for magnetoacoustic waves in an isothermal solar atmosphere permeated by an uniform vertical magnetic field. This expression derived by Roberts (2006) is given by

$\omega_{\mathrm{C} 4}=c_{\mathrm{t}}\left[\frac{1}{4 H_{\mathrm{p}}^{2}}\left(\frac{c_{\mathrm{t}}}{c_{\mathrm{s}}}\right)^{4}-\frac{1}{2} \gamma g \frac{\partial}{\partial z}\left(\frac{c_{\mathrm{t}}^{2}}{c_{\mathrm{s}}^{4}}\right)+\frac{1}{v_{\mathrm{A}}^{2}}\left(N^{2}+\frac{g}{H_{\mathrm{p}}} \frac{c_{\mathrm{t}}^{2}}{c_{\mathrm{s}}^{2}}\right)\right]^{1 / 2}$,

where $N^{2}$ is the squared Brunt-Väisälä frequency, $c_{\mathrm{t}}=c_{\mathrm{s}} v_{\mathrm{A}} /$ $\sqrt{c_{\mathrm{s}}^{2}+v_{\mathrm{A}}^{2}}$ is the cusp speed, $\gamma$ is the adiabatic index, and $g$ is the gravity. 


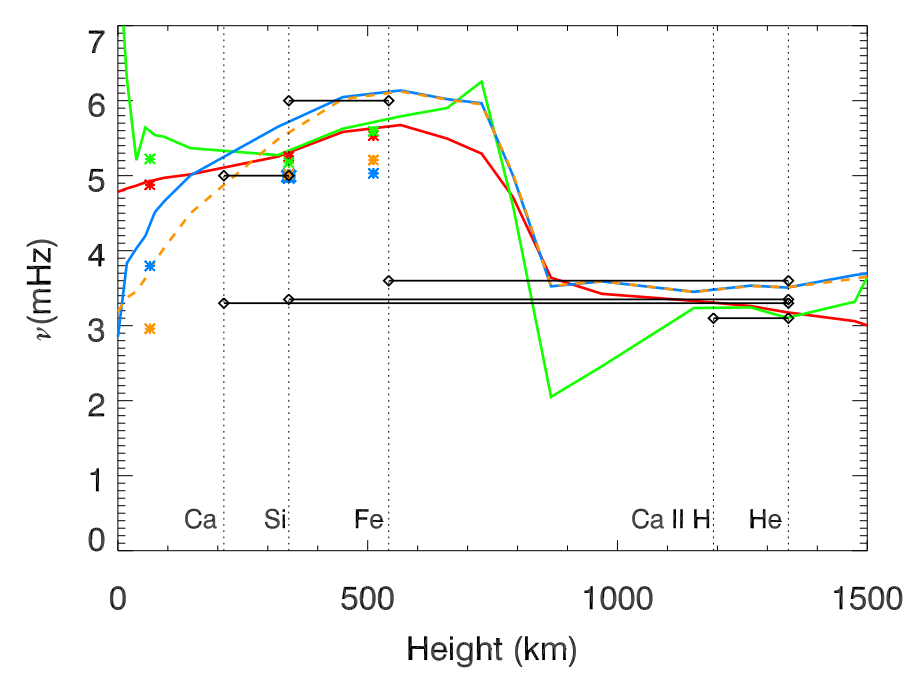

Fig. 12. Variation of the cutoff frequency in the umbral atmosphere Color lines represent the cutoff values computed for Avrett (1981) model and the color asterisks correspond to the cutoffs calculated for the inverted umbral atmosphere. The color indicates the formula used: red (Eq. (1)), green (Eq. (2)), blue (Eq. (3)), and orange (Eq. (4)). Horizontal black lines with diamonds indicate the observational cutoffs as determined from Figs. 6-11 (see Table 1). The black lines cover the range of heights between the formation height of the two lines used for each measurement. Vertical dotted lines indicate the formation height of the spectral lines as determined from the analysis of wave propagation.

Our approach to measuring the cutoff is slightly different from that performed by Wiśniewska et al. (2016). First, we estimated the phase shift between various combinations of pairs of lines, instead of only between the line with highest formation height and the rest. This way, we can probe localized layers of the atmosphere and restrict the derived cutoff to a more constrained height. Second, in addition to the analysis of the phase difference, we complemented the determination of the cutoff with the amplification spectra.

The results are illustrated in Fig. 12. We computed the analytical cutoff using Eqs. (1)-(4) for the atmospheric values retrieved from the inversions of the Ca I $10839 \AA$ and Si I $10827 \AA$ lines. They are represented by red, green, blue, and orange asterisks, respectively. The height of these data is chosen at the maximum of the response function of the Ca I $10839 \AA$, Si I $10827 \AA$, and Fe I $5435 \AA$ lines. The analytical cutoffs of the former were obtained from the inversions of the Ca I $10839 \AA$ line, while the other two were computed from the stratification retrieved from the Si I $10827 \AA$ inversion. We note that the sensitivity of this inversion to the formation height of the Fe I $5435 \AA$ line is low.

The observational cutoffs from Sect. 3.2 are indicated by black horizontal lines. They link the two atmospheric heights (indicated by diamonds) used for computing the corresponding phase and amplification spectra. In these cases, the formation heights are determined based on the analysis of the wave propagation. The formation height of the Si I $10827 \AA$ line has been set to the height given by the response function (same height from the previous paragraph), while the rest of the formation heights are derived from the height differences indicated in Table 1. The difference in formation height between the Si I $10827 \AA$ and Fe I $5435 \AA$ lines obtained from the analysis of the oscillations is consistent with that retrieved from the evaluation of their response functions. On the contrary, the peak of the response function of the Ca I $10839 \AA$ line is deeper than the location expected from the phase shift of its oscillations with respect to those from the Si I $10827 \AA$ line.

By plotting the measured cutoff over a range of heights (black horizontal lines), we explicitly indicate that the atmospheric layer with that value as local cutoff frequency is undetermined. The observational cutoffs must be interpreted as the minimum value of the actual cutoff between the two heights used in each measurement. However, thanks to the use of pairs of lines with close formation heights and the combination of many spectral lines, some conclusions about the stratification of the cutoff frequency can be extracted: (i) at the deep photosphere, its value is around $5 \mathrm{mHz}$, as shown by the analysis of the line pair Ca I $10839 \AA$-Si I $10827 \AA$; (ii) the cutoff increases at higher photospheric layers, since the line pair Si I 10827 $\AA$-Fe I $5435 \AA$ exhibits a value around $6 \mathrm{mHz}$; and (iii) at the chromosphere the cutoff is significantly lower, around $3.1 \mathrm{mHz}$. The later is proven by the analysis of the pair of chromospheric lines Ca II H-He I $10830 \AA$ and by the cutoff estimated from the Ca I $10839 \AA$ - He I $10830 \AA$ and Si I $10827 \AA-H e$ I $10830 \AA$ pairs. Since we concluded that from the deep photosphere to the high photosphere the cutoff is higher than $5 \mathrm{mHz}$, the measured phase difference for frequencies around $3.3 \mathrm{mHz}$ must be produced at higher layers.

Following Wiśniewska et al. (2016), we compared the observational results of the cutoff with the values computed using various formulae from the literature in a standard model (in our case, the umbral atmosphere from Avrett 1981). The four calculated cutoffs show some common properties that are confirmed by our observational measurements. The cutoff increases from the base of the photosphere to higher layers (up to $\sim 600-700 \mathrm{~km}$, depending on the formula), while at the chromosphere it is much lower, that is, slightly above $3 \mathrm{mHz}$. The Roberts (2006) cutoff expression (Eq. (4)) for magnetoacoustic waves is almost indistinguishable from Schmitz \& Fleck (1998) formula (Eq. (3)) from the high photosphere to higher layers. The chromospheric cutoffs $\omega_{\mathrm{C} 1}$ and $\omega_{\mathrm{C} 2}$ show a remarkable agreement with the observational values, assuming that Avrett (1981) model describes the umbra of this sunspot. At the photosphere, all the cutoff values calculated from Avrett (1981) model and from the inversions (asterisks, using the same analytical expressions) exhibit some discrepancies with the observations. The cutoff retrieved from the inversions using Roberts (2006) formula (orange asterisks) shows a perfect match at the formation height of the Si I $10827 \AA$ line, although it does not reproduce the higher value observed at the high photosphere from the analysis of the Si I $10827 \AA$ and Fe I $5435 \AA$ pair of lines.

The presence of cutoff frequencies implies that waves can be trapped in the solar atmosphere owing to wave reflections and a cavity can potentially be formed (Schmitz \& Fleck 1992). The phase relations between the velocity and intensity can be used to determine whether the oscillations are propagating waves or standing waves (e.g., Fujimura \& Tsuneta 2009). In an adiabatic case, the phase shift between intensity and Doppler velocity (with positive values corresponding to redshifts) for propagating waves is $180^{\circ}$, while standing modes show a phase difference of $90^{\circ}$ (e.g., Deubner 1974; Al et al. 1998). A phase shift of $90^{\circ}$ indicates that the intensity signal is delayed a quarter period with respect to the velocity signal. Figure 13 illustrates the temporal evolution of the velocity and core intensity oscillations of the He I $10830 \AA$ filtered in the frequency band between $4.5 \mathrm{mHz}$ and $8 \mathrm{mHz}$ and averaged for all the spatial locations inside the umbra. The core intensity is defined as the intensity of the minimum from a second order polynomial fit to a spectral region 


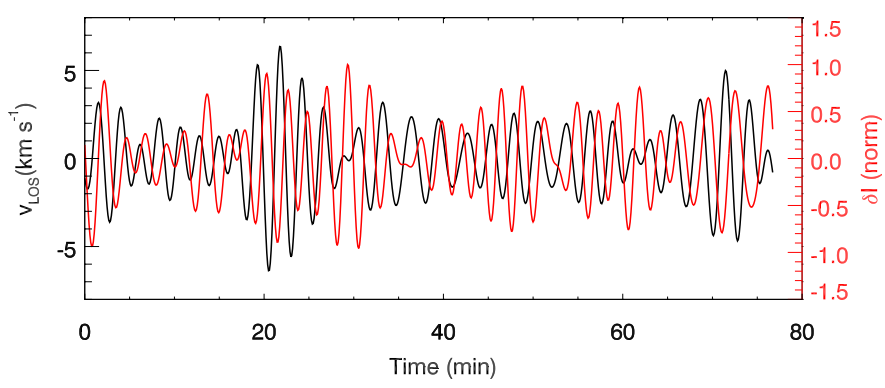

Fig. 13. Temporal evolution of the LOS velocity (black line) and core intensity (red line) of the He I $10830 \AA$ filtered in the $4.5-8 \mathrm{mHz}$ frequency range and spatially averaged for all the umbral spatial locations.

of $0.23 \AA$ around the core of the line. Although the $4.5-8 \mathrm{mHz}$ bandpass corresponds to propagating waves, according to the cutoff values observationally determined and plotted in Fig. 12, during most of the temporal series illustrated in Fig. 13 the intensity signal trails the velocity signal, as expected for standing waves. On the contrary, during some temporal spans they have opposite phases (for example, around $t=45 \mathrm{~min}$ ), as theory predicts for propagating waves. The spatially average phase shift over the whole temporal series between velocity and intensity is $108^{\circ} \pm 14^{\circ}$, which departs from the $90^{\circ}$ predicted for standing waves. This is consistent with the phase shift measured between the velocity from two chromospheric lines (Fig. 11), which proves that there is upward wave propagation at chromospheric heights. We interpret that slow magnetoacoustic waves are partially reflected at the transition region, forming a chromospheric standing wave (Fleck \& Deubner 1989), while the rest of the slow mode wave energy is propagating in the upward direction (Jess et al. 2012).

\section{Conclusions}

We have evaluated the cutoff frequency in a sunspot umbra and its variation with the atmospheric height by analyzing the phase difference and amplification spectra between several pairs of spectral lines that probe various layers. Our measurements show that between the deep photosphere and high photosphere the cutoff frequency increases from $5 \mathrm{mHz}$ to $6 \mathrm{mHz}$. At higher chromospheric values the cutoff is reduced to $\sim 3.1 \mathrm{mHz}$. These results have been compared with the values obtained from the application of several analytical cutoff forms to the atmosphere inferred from the inversion of the photospheric lines and to a standard model of umbral stratification. This comparison reveals some significant differences at the photosphere.

Our study has strong implications on our understanding of wave propagation in magnetized structures and its applications to several topics of solar research. Sunspots are one of the main targets of local helioseismology. One of the key measurements of these techniques is the travel time of $p$-mode waves between two locations on the solar surface, generally filtered in frequency and phase speed. The travel-time shift is then defined as the difference between the travel time measured in a region of interest (for example, a sunspot) and that measured in a quiet Sun region. Recent works have found that the cutoff frequency is a main contributor to travel-time shift measurements (Lindsey et al. 2010; Schunker et al. 2013; Felipe et al. 2017). Because of the Wilson depression, the height of the upper turning point of the waves (that is, the height where the wave frequency equals the local cutoff frequency) is lowered. Thus, waves beneath sunspots travel a shorter path than in quiet Sun regions, and their travel time is reduced. Interpretation of the travel-time shifts can potentially benefit from simultaneous estimations of the atmospheric cutoff frequencies, such as those carried out in this work. These data would complement the helioseismic measurements and would help to define the solar layers sampled by the $p$-modes at each frequency.

Compressive waves are also one of the proposed mechanisms to explain chromospheric heating (e.g., Bello González et al. 2010b; Felipe et al. 2011; Kanoh et al. 2016; Krishna Prasad et al. 2017). These studies usually quantify the available energy flux of the propagating waves at photospheric heights and, thus, a detailed characterization of the cutoff is fundamental to estimate which frequencies can actually contribute to the heating.

Acknowledgements. We thank Dr. N. Bello González for her support during the observations. Financial support from the Spanish Ministry of Economy and Competitivity through projects AYA2014-55078-P, AYA2014-60476$\mathrm{P}$, and AYA2014-60833-P is gratefully acknowledged. C.K. was supported in part by grant DE 787/5-1 of the German Science Foundation (DFG). The $1.5 \mathrm{~m}$ GREGOR solar telescope was built by a German consortium under the leadership of the Kiepenheuer-Institut für Sonnenphysik in Freiburg with the LeibnizInstitut für Astrophysik Potsdam, the Institut für Astrophysik Göttingen, and the Max-Planck-Institut für Sonnensystemforschung in Göttingen as partners, and with contributions by the Instituto de Astrofísica de Canarias and the Astronomical Institute of the Academy of Sciences of the Czech Republic.

\section{References}

Al, N., Bendlin, C., \& Kneer, F. 1998, A\&A, 336, 743

Avrett, E. H. 1981, in The Physics of Sunspots, eds. L. E. Cram, \& J. H. Thomas, 235

Bel, N., \& Leroy, B. 1977, A\&A, 55, 239

Bello González, N., Flores Soriano, M., Kneer, F., Okunev, O., \& Shchukina, N. 2010a, A\&A, 522, A31

Bello González, N., Franz, M., Martínez Pillet, V., et al. 2010b, ApJ, 723, L134

Bloomfield, D. S., Lagg, A., \& Solanki, S. K. 2007, ApJ, 671, 1005

Cally, P. S. 2006, Roy. Soc. London Philos. Trans. Ser. A, 364, 333

Cally, P. S. 2007, Astron. Nachr., 328, 286

Campos, L. M. B. C. 1986, Rev. Mod. Phys., 58, 117

Centeno, R., Collados, M., \& Trujillo Bueno, J. 2006, ApJ, 640, 1153

Collados, M. 1999, in Third Advances in Solar Physics Euroconference: Magnetic Fields and Oscillations, eds. B. Schmieder, A. Hofmann, \& J. Staude, ASP Conf. Ser., 184, 3

Collados, M. V. 2003, in Polarimetry in Astronomy, ed. S. Fineschi, Proc. SPIE, 4843, 55

Collados, M., Martinez Pillet, V., Ruiz Cobo, B., del Toro Iniesta, J. C., \& Vazquez, M. 1994, A\&A, 291, 622

Collados, M., López, R., Páez, E., et al. 2012, Astron. Nachr., 333, 872

De Moortel, I., Ireland, J., Hood, A. W., \& Walsh, R. W. 2002, A\&A, 387, L13

De Pontieu, B., Erdélyi, R., \& James, S. P. 2004, Nature, 430, 536

Deubner, F.-L. 1974, Sol. Phys., 39, 31

Deubner, F.-L., \& Gough, D. 1984, ARA\&A, 22, 593

Felipe, T., Khomenko, E., Collados, M., \& Beck, C. 2010, ApJ, 722, 131

Felipe, T., Khomenko, E., \& Collados, M. 2011, ApJ, 735, 65

Felipe, T., Braun, D. C., \& Birch, A. C. 2017, A\&A, 604, A126

Fleck, B., \& Deubner, F.-L. 1989, A\&A, 224, 245

Fujimura, D., \& Tsuneta, S. 2009, ApJ, 702, 1443

Giovanelli, R. G., Livingston, W. C., \& Harvey, J. W. 1978, Sol. Phys., 59,49

Hammer, R., Musielak, Z. E., \& Routh, S. 2010, Astron. Nachr., 331, 593

Heggland, L., Hansteen, V. H., De Pontieu, B., \& Carlsson, M. 2011, ApJ, 743, 142

Hofmann, A., Arlt, K., Balthasar, H., et al. 2012, Astron. Nachr., 333, 854 Jefferies, S. M., McIntosh, S. W., Armstrong, J. D., et al. 2006, ApJ, 648, L151 Jess, D. B., De Moortel, I., Mathioudakis, M., et al. 2012, ApJ, 757, 160 Kanoh, R., Shimizu, T., \& Imada, S. 2016, ApJ, 831, 24

Khomenko, E., \& Collados, M. 2006, ApJ, 653, 739

Khomenko, E., Centeno, R., Collados, M., \& Trujillo Bueno, J. 2008, ApJ, 676, L85

Krishna Prasad, S., Jess, D. B., Van Doorsselaere, T., et al. 2017, ApJ, 847, 5 Kuckein, C., Martínez Pillet, V., \& Centeno, R. 2012, A\&A, 542, A112 
Kuckein, C., Denker, C., Verma, M., et al. 2017, in Fine Structure and Dynamics of the Solar Atmosphere, eds. S. Vargas Domínguez, A. G. Kosovichev, P. Antolin, \& L. Harra, IAU Symp., 327, 20

Lamb, H. 1909, Proc. London. Math. Soc, 7, 122

Lindsey, C., Cally, P. S., \& Rempel, M. 2010, ApJ, 719, 1144

Lites, B. W., Rutten, R. J., \& Kalkofen, W. 1993, ApJ, 414, 345

Löfdahl, M. G. 2002, in Image Reconstruction from Incomplete Data, eds. P. J.

Bones, M. A. Fiddy, \& R. P. Millane, Proc. SPIE, 4792, 146

Löhner-Böttcher, J., Bello González, N., \& Schmidt, W. 2016, Astron. Nachr., 337,1040

Martínez Pillet, V., Lites, B. W., \& Skumanich, A. 1997, ApJ, 474, 810

McIntosh, S. W., \& Jefferies, S. M. 2006, ApJ, 647, L77

Moradi, H., \& Cally, P. S. 2008, Sol. Phys., 251, 309

Murawski, K., \& Musielak, Z. E. 2010, A\&A, 518, A37

Musielak, Z. E., Musielak, D. E., \& Mobashi, H. 2006, Phys. Rev. E, 73, 036612

Orrall, F. Q. 1966, ApJ, 143, 917

Perera, H. K., Musielak, Z. E., \& Murawski, K. 2015, MNRAS, 450, 3169

Puschmann, K. G., Denker, C., Kneer, F., et al. 2012, Astron. Nachr., 333, 880

Rae, I. C., \& Roberts, B. 1982, ApJ, 256, 761

Roberts, B. 1983, Sol. Phys., 87, 77

Roberts, B. 2006, Phil. Trans. R. Soc. London, Ser. A, 364, 447

Routh, S., Musielak, Z. E., \& Hammer, R. 2013, ApJ, 763, 44
Ruiz Cobo, B., \& del Toro Iniesta, J. C. 1992, ApJ, 398, 375

Schmidt, W., von der Lühe, O., Volkmer, R., et al. 2012, Astron. Nachr., 333, 796

Schmitz, F., \& Fleck, B. 1992, A\&A, 260, 447

Schmitz, F., \& Fleck, B. 1998, A\&A, 337, 487

Schmitz, F., \& Fleck, B. 2003, A\&A, 399, 723

Schunker, H., \& Cally, P. S. 2006, MNRAS, 372, 551

Schunker, H., Gizon, L., Cameron, R. H., \& Birch, A. C. 2013, A\&A, 558, A 130

Socas-Navarro, H. 2001, in Advanced Solar Polarimetry - Theory, Observation, and Instrumentation, ed. M. Sigwarth, ASP Conf. Ser., 236, 487

Stark, B. A., \& Musielak, Z. E. 1993, ApJ, 409, 450

Thomas, J. H. 1982, ApJ, 262, 760

Thomas, J. H. 1983, Ann. Rev. Fluid Mech., 15, 321

Tziotziou, K., Tsiropoula, G., Mein, N., \& Mein, P. 2006, A\&A, 456, 689

van Noort, M., Rouppe van der Voort, L., \& Löfdahl, M. G. 2005, Sol. Phys., 228,191

Vecchio, A., Cauzzi, G., Reardon, K. P., Janssen, K., \& Rimmele, T. 2007, A\&A, 461, L1

Wiśniewska, A., Musielak, Z. E., Staiger, J., \& Roth, M. 2016, ApJ, 819, L23

Yuan, D., Sych, R., Reznikova, V. E., \& Nakariakov, V. M. 2014, A\&A, 561, A19 